\title{
Modelo de intervención para competencias digitales del Programa Bibliotecas Públicas de Aguascalientes
}

\author{
José Luis González Sandoval* \\ Francisco Javier Álvarez-Rodríguez ${ }^{\star *}$ \\ Jaime Muñoz Arteaga**
}

Artículo recibido:

22 de agosto de 2018

Artículo aceptado:

10 de enero de 2019

Artículo de investigación

\section{Resumen}

El presente artículo describe, de manera general, un modelo de intervención para el desarrollo de la sociedad del conocimiento del estado de Aguascalientes, particularmente enfocado en las bibliotecas públicas en las que se aplicaron estrategias para capacitar al personal bibliotecario que labora en las unidades de información. En dicha intervención, se describen los cursos, objetivos y logística, así como los resultados que se obtuvieron, con la finalidad de fortalecer las competencias digitales del personal bibliotecario, además de las etapas que se cubrieron en dicho proceso. El modelo se justifica y adquiere valor por sus

\footnotetext{
* Departamento de Información Bibliográfica, Universidad Autónoma de Aguascalientes, México basset137@gmail.com ** Centro de Ciencias Básicas, Universidad Autónoma de Aguascalientes, México fjalvar.uaa@gmail.com_jmauaa@gmail.com 
características de flexibilidad, adaptabilidad e interdisciplinariedad de cursos, especialmente diseñados para reducir la brecha digital en los bibliotecarios y en la sociedad. Se exponen también en los siguientes apartados el contexto e importancia de las habilidades y competencias obtenidas a lo largo del programa, así como datos cualitativos; más aún, esta propuesta permitió analizar las condiciones del sistema bibliotecario de la entidad y conocer las características de infraestructura que posee.

Palabras clave: Modelo de Intervención; Bibliotecas Públicas; Habilidades Informativas; Brecha Digital.

Intervention model for digital competences of Aguascalientes's Public Libraries Program

José Luis González Sandoval, Francisco Javier ÁlvarezRodríguez and Jaime Muñoz Arteaga

\section{Abstract}

The present article describes, in a general way, an intervention model for the development of the knowledge society of Aguascalientes's State, particularly focused on the public libraries where strategies were applied to train the library staff who works in the information units. This intervention describes the courses, objectives, logistics and results obtained in order to strengthen the digital skills of the library staff, as well as the stages covered by this process. The model is justified and acquires value given its characteristics of flexibility, adaptability and the interdisciplinarity of these courses, specially designed to reduce the digital divide in librarians and in the society. The context and importance of the skills and competences obtained throughout the program, as well as the qualitative data, are also discussed in the following sections; furthermore, the proposal has allowed to analyze the library system's condition of that entity and to meet the characteristics of infrastructure that it possesses.

Keywords: Intervention Model; Public Libraries; Information Skills; Digital Divide. 


\section{INTRODUCCIÓN}

Z1 presente documento expone el trabajo que se realizó en el estado Ede Aguascalientes para implementar un modelo de intervención, con el objetivo de desarrollar competencias digitales en los bibliotecarios que atienden cada una de las unidades de información, mostrando cuáles fueron sus etapas, cómo fue aplicado y cuáles fueron los resultados que se obtuvieron. Más aún, este artículo presenta un panorama general de las bibliotecas públicas, cuál es su función y relación con la sociedad que atiende, pero también, por qué están consideradas como un eslabón entre la información, el conocimiento y la sociedad que requiere de estos servicios.

La experiencia que aquí se presenta está plasmada en varios apartados en los que se describen los antecedentes de las bibliotecas públicas, la problemática a la que se enfrentan, así como una descripción del modelo de intervención presentado, su aplicación en el entorno de bibliotecas, para dar paso a la confirmación de la validación, mostrando cada una de las etapas en el proceso de implementación. Finalmente, se muestran y describen los resultados obtenidos desde la planeación, así como de la implementación de dicho modelo.

\section{Antecedentes de las bibliotecas públicas}

De acuerdo con las directrices que marca la Federación Internacional de Asociaciones de Bibliotecarios y Bibliotecas IFLA/Unesco, aparecidas en 1986, el papel de la biblioteca deberá ir evolucionando de acuerdo con las nuevas necesidades de la sociedad y de su entorno, el cual se torna cada vez más especializado y pleno de información. Ahora bien, para que un individuo se considere un ciudadano digital tendrá que adquirir, poseer y manejar ciertas habilidades y competencias que le permitan desenvolverse en la sociedad del conocimiento, para que formen parte de esta nueva era, denominada sociedad de la información y sociedad del conocimiento.

En la introducción de las directrices publicadas en 2000 por el Consejo Nacional para la Cultura y las Artes (Conaculta), la Unesco y la IFLA, Phillip Gill escribía: 
Las bibliotecas públicas tienen ante sí una apasionante oportunidad de ayudar a que todos tengan acceso al intercambio mundial del que antes se hablaba y a salvar lo que se ha dado en llamar "la brecha digital". Pueden conseguirlo dando al público acceso a la tecnología de la información, enseñando nociones elementales de informática y participando en programas para combatir el alfabetismo (Gill, 2002: 20).

Ante esta aseveración, se concibe a la biblioteca como un eslabón que se encargará de proporcionar fuentes informativas en cualquier formato, ya sea en papel o digital, además de proporcionar servicios para ayudar a sus usuarios y comunidades en aspectos educativos informales, recreativos artísticos, culturales (IFLA, 1994) y, por lo tanto, intelectuales, para disminuir las carencias de los usuarios de la información, y así reducir la brecha digital en la sociedad, basándose en las competencias informativas (Cortés et al., 2012: 3).

\section{Descripción de la Red Estatal de Bibliotecas Públicas}

La Red Estatal de Bibliotecas Públicas del Estado de Aguascalientes es administrada por el Instituto Cultural de Aguascalientes (ICA). Aquí encontramos, de forma gratuita, diversos servicios y recursos bibliográficos disponibles para toda la población. Existen 66 bibliotecas públicas, las cuales cuentan con un total de 401,476 volúmenes, todas con módulo de servicios digitales, donde se atiende cada año en promedio a 142,441 usuarios, 17 con taller de computación, de donde se han atendido a sesenta y tres mil niños y jóvenes.

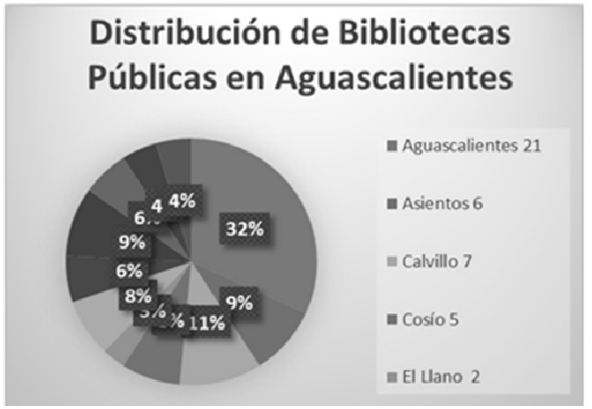

Gráfica 1. Distribución (por municipios) de las bibliotecas públicas en el estado de Aguascalientes. Fuente: Elaboración propia.

En la Gráfica 1 se observa cuántas bibliotecas hay por municipio y el porcentaje que representan en cada cual, por ejemplo, en el municipio de Aguascalientes hay 21 unidades de información, las cuales representan el 
32 por ciento, lo que indica que en la capital del estado es donde se encuentra el mayor número de bibliotecas y, por lo tanto, el mayor número de bibliotecarios. Por otra parte, el municipio con menos bibliotecas es El Llano, con sólo dos bibliotecas.

En este trabajo se analizan, como casos de estudio, sólo tres bibliotecas, a las cuales se considera las más importantes tanto por su ubicación geográfica, como su infraestructura y su acervo bibliográfico (Esquema 1):

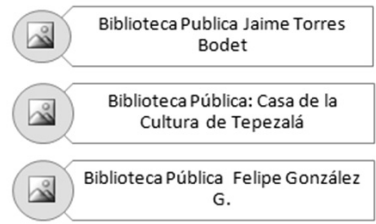

Esquema 1. Bibliotecas públicas de Aguascalientes que se visitaron. Fuente: Elaboración propia.

Las bibliotecas mencionadas se eligieron por diferentes aspectos: en el caso de la Biblioteca pública "Torres Bodet", hay que mencionar que es la unidad de información más grande que administra el sistema de bibliotecas y que tiene un acervo con diversidad de colecciones, una amplia gama de servicios para atención al público, una librería, aulas y auditorios, además de que cuenta con el mayor número de personal.

En el caso de la Biblioteca de la Casa de la Cultura de Tepezalá se eligió por ser una de las más alejadas de la capital del estado y, por otra parte, con la finalidad de conocer y observar el funcionamiento de los diversos programas y servicios que ahí se han implementado.

Finalmente, en el caso de la Biblioteca "Felipe González", se eligió por ser la que más recientementese restauró y equipó con salas de cómputo, de trabajo, de lectura, multiusos y auditorios, así como programas de atención a usuarios; de ahí que fueran bibliotecas que estaban funcionando al cien por ciento de su capacidad.

\section{Problemática de las bibliotecas públicas de Aguascalientes}

De acuerdo con la información que se encuentra en la página de Gobierno del Estado, en estas bibliotecas observamos que, en su estructura organizacional, se cuenta con los siguientes servicios: salas de lectura, conexión 
a Internet (o módulo de servicios digitales), talleres de computación para adultos y niños, visitas guiadas, servicio de fotocopias, fomento a la lectura, préstamo de libros en sala y a domicilio, por lo que se corrobora que el estado actual de las bibliotecas es básico y suficiente, ya que la infraestructura es adecuada para atender a cerca de sesenta y tres mil niños y un promedio de 142,441 usuarios promedio (GEA, 2016). Sin embargo, un problema en esta localidad es que no se cuenta con personal bibliotecario capacitado, menos aun profesional, en el área de la información; ni con las habilidades y competencias para enfrentar los retos actuales para un bibliotecario del siglo XXI, menos aun las de un ciudadano digital (Area, 2015: 2).

Cabe mencionar que, aunque se cuenta con espacios de infraestructura, equipamiento, conectividad, acervos físicos, acervos digitales, colecciones y actividades enfocadas a su comunidad, al personal que atiende no se le puede considerar como profesionales de la información, por lo que la principal problemática es la falta de capacitación del personal bibliotecario.

Parte de esta problemática se constata con los resultados obtenidos en el documento: "Instrumento de diagnóstico para personal y auxiliares que laboran en bibliotecas estatales", ${ }^{1}$ instrumento que es parte de una encuesta para conocer las habilidades y competencias que tenía el personal bibliotecario. En la Gráfica 2 se observan los resultados obtenidos, gracias al proyecto Fomix-Ags. 2011-C01-171877:

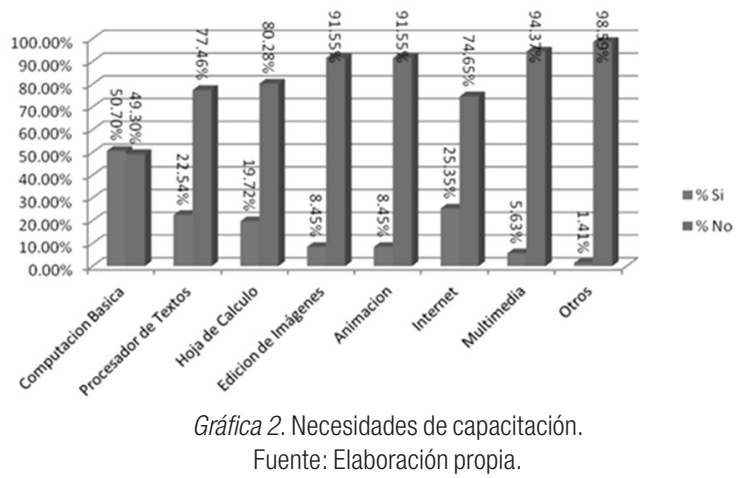

Como se observa, el personal de biblioteca sólo ha tenido un acercamiento en temas de computación básica, pero en programas como animación o programas multimedia, hoja de cálculo o edición de imágenes, el porcentaje 
es muy bajo, por lo que estos indicadores confirman una carencia en cuanto al dominio y conocimiento de esos programas.

\section{Modelo de intervención De COMPETENCIAS Digitales}

Un modelo de intervención se podría definir como un conjunto de elementos que tienen la finalidad de desarrollar competencias y habilidades (Dehesa et al., 2015: 72) que permiten al ciudadano apropiarse de las tic o de las nuevas tecnologías, desde un plano básico, y que se requieren para su desarrollo intelectual y sociocultural (Area, 2002: 7).

El modelo de intervención que se describe enseguida tiene características muy particulares que le permiten solucionar las necesidades que afectan los problemas derivados de la brecha digital. En primer lugar, se puede mencionar que es un modelo basado para desarrollarse en ambientes formales e informales de aprendizaje, enfocados para atender a la sociedad en general, entre las que se encuentran personas con diferentes niveles de educación, desempleados, así como adultos de la tercera edad que pertenecen a diferentes estratos sociales (entre otros, profesores, profesionistas y ciudadanos de la capital de estado, sus municipios y sus comunidades sus zonas indígenas y rurales).

Ahora bien, el modelo está enfocado en desarrollar la alfabetización digital, en la que sus principales actores son el gobierno estatal, las instituciones de educación superior y las empresas con infraestructura que permite implementar contenidos digitales basados en las tic.

Ante estos elementos, el modelo abarcará los aspectos de contenidos, capacitación, equipamiento, conectividad y certificación, para dar paso a la ciudadanía y, por consiguiente, al ciudadano digital de Aguascalientes (Esquema 2):

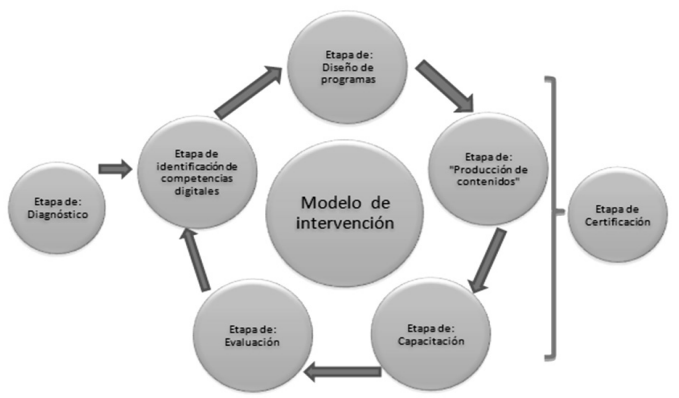

Esquema 2. Modelo de intervención. Fuente: Elaboración propia. 
Para solucionar esta problemática, el modelo de intervención se justifica con los siguientes argumentos: el primero es que se proponen soluciones directas a cada una de las limitantes que se encontraron, tanto en la estructura de bibliotecas, así como en su funcionamiento; por ejemplo, se les proporcionó equipamiento de cómputo por parte de las autoridades estatales y se dio conectividad a Internet, también se hizo énfasis en fortalecer las habilidades y las competencias informativas del personal bibliotecario para afrontar los retos que la sociedad demanda en cada una de las unidades de información.

Un segundo argumento se fundamenta en que los cursos propuestos están enfocados y relacionados con los servicios digitales, propios de una sociedad de la información, es decir; entendiendo que los servicios de las bibliotecas y la información están evolucionando hacia una era digital, entonces, el proceso de enseñanza y aprendizaje también deberá irse adaptando a una función didáctica, para dar pie a la alfabetización informativa, según Torres et al. (2002: 449), en la que se enfocarán los esfuerzos en desarrollar habilidades y competencias que permitan a los profesionales de la información entender su entorno actual, así se podrá adaptar y evolucionar hacia la era digital propia del siglo XXI.

En este caso, se desarrollaron cursos que les permitieran utilizar herramientas de búsqueda de información, navegadores de Internet, procesadores de palabras, uso y manejo de bases de datos especializadas, portales digitales, base de datos bajo la iniciativa de Open Access, redes sociales, así como el uso y manejo de aulas virtuales.

\section{Antecedentes del modelo de intervención}

Un fenómeno que se ha desarrollado en las últimas décadas en nuestro entorno económico, académico y cultural, y en todas las áreas del acontecer social es la llamada brecha digital, que apunta a un problema que se presenta entre los individuos y sus comunidades que utilizan las tecnologías de la comunicación y la información (TIC) y entre las personas que no tienen acceso a esos mismos servicios por estos mismos medios.

De forma general, el modelo de intervención que se plantea está fundamentado (y enfocado) para generar y plantear una estrategia que ayude a disminuir la brecha digital en el sistema de bibliotecas públicas y que se logre 
un doble beneficio, tanto en sus bibliotecarios, como en la sociedad a las que se atiende. Por lo tanto, el modelo propone un desarrollo de competencias y habilidades digitales que ayuden a incluir el uso de las tic en los bibliotecarios y, a su vez, en los ciudadanos que se puedan adaptar a la sociedad del conocimiento, como lo apuntan Muñoz et al. (2014: 128) y, a su vez, se pueda lograr por parte de los individuos una ciudadanía digital, ya que también se ha propuesto un modelo de individuo digital, capaz de integrar herramientas y servicios en su contexto sociocultural, que lo ayudarán a integrarse y adaptarse a la sociedad del siglo XXI. De acuerdo con los dominios que Álvarez et al. (2015:3) (Esquema 3) proponen:

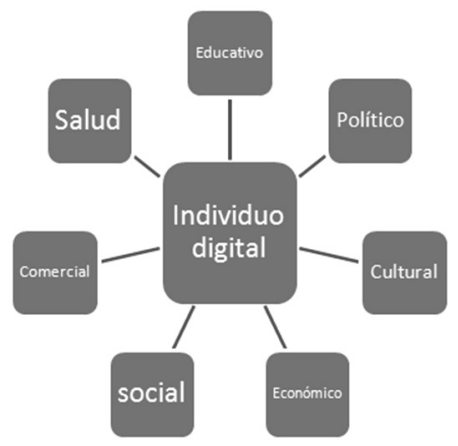

Esquema 3. Dominios del individuo digital. Fuente: Álvarez et al. (2015).

Estructura y operación del modelo de bibliotecas públicas

Para sustentar el modelo de intervención en las bibliotecas públicas, se planeó una serie de cursos de capacitación adaptados a su estructura y que se reflejara en una mejoría en los servicios que se ofrecen, además de que tuvieran una relación con la forma de operar en cada una de sus bibliotecas, todo esto con la finalidad de que los participantes adquirieran y desarrollaran habilidades profesionalizantes que les permita involucrarse con la sociedad a la que atienden y les permitieran aportar ideas desde cada una de sus bibliotecas.

De este modo, con base en los resultados de la encuesta aplicadas con anterioridad, se consideró que era pertinente abarcar dos grandes ramas: 1) la enfocada a las tecnologías de la información y su aplicación, por medio de las TIC, ya que era necesario actualizar al personal en relación con las aplicaciones que se pueden utilizar de forma práctica y con la infraestructura que se 
cuenta; 2) se determinó desarrollar el propio perfil del bibliotecario, es decir, se impartieron cursos que vincularan las actividades propias de un bibliotecario que cataloga, almacena, clasifica y propone cursos para crear un vínculo entre el profesional de la información y los servicios, las herramientas y las bondades que las aplicaciones de las TIC ofrecen a su comunidad.

En cuanto a estos aspectos tecnológicos, en un primer nivel se incluyeron las competencias básicas que ayudarían al bibliotecario a desarrollar habilidades de manejo de cómputo (léase partes de una computadora, sistema operativo, manejo de procesadores de palabras), involucrarse con las herramientas propias que ofrece la Internet, por ejemplo, los motores de búsqueda, los navegadores, o bien los correos electrónicos.

En el nivel intermedio, se enfocó a la profesionalización con cursos relacionados con el comercio y comunicación digital, así como usar y modificar archivos en diferentes formatos, protección digital, descargar e instalar programas de cómputo (incluido el aprendizaje y uso del software libre a disposición de cualquier persona inmersa en el manejo de información); cabe mencionar que también se impartieron cursos para editar imágenes o implementar la seguridad en la Internet.

En el aspecto bibliotecario, se profesionalizaron en cursos cuya intención era el desarrollo de las habilidades propias de un bibliotecario del siglo XXI; en este caso se determinó que habría que enfatizar en las estrategias de búsqueda de información, en la micreoenseñanza, incluyendo aspectos que están inmersos en la publicación de material bibliohemerográfico (impreso o digital), ya fueran las licencias Creative Commons, o los derechos de autor; o bien la llamada iniciativa de Open Access que se presenta de manera muy particular en la producción de información académica y especializada, la cual forma parte de las bibliotecas.

\section{Impartición de cursos}

De acuerdo con la problemática antes expuesta, se pusieron en marcha diferentes cursos, especialmente diseñados para contrarrestar el rezago en las habilidades y competencias del personal bibliotecario, con la finalidad de afrontar nuevos retos que se presentan en cada una de las unidades de información distribuidas en el estado. Ahora bien, los cursos desarrollaron las áreas de “Tecnologías básicas”, complementando con cursos enfocados en "Competencias profesionalizantes del perfil bibliotecario", lo cual permitió capacitar a los bibliotecarios, de manera introductoria, en la conectividad a Internet, 
así como en aspectos especializados de tecnología digital; igualmente se desarrollaron proyectos enfocados en necesidades particulares de cada una de sus comunidades y en sus unidades de información (Cuadro 1):

\begin{tabular}{|c|c|c|c|}
\hline \multicolumn{2}{|c|}{ Tecnologías básicas } & \multicolumn{2}{|c|}{ Competencias básicas profesionalizantes } \\
\hline $\begin{array}{l}\text { Tecnologías de } \\
\text { la información }\end{array}$ & $\begin{array}{l}\text { - } \text { Navegador de Internet } \\
\text { - } \text { Motores de búsqueda } \\
\text { - } \text { gestión de la Información } \\
\text { - } \text { Correo electrónico } \\
\text { - Opciones interactivas } \\
\text { digitales } \\
\text { - Búsqueda avanzada en } \\
\text { Internet } \\
\text { - Seguridad en Internet } \\
\text { - E-comunicación } \\
\text { - E-comercio } \\
\text { - Uso y modificación de } \\
\text { archivos existentes }\end{array}$ & $\begin{array}{l}\text { - Profesionalizantes en } \\
\text { bibliotecología } \\
\text { - Habilidades docentes }\end{array}$ & $\begin{array}{ll}\text { - } & \text { Derechos } \\
& \text { de autor } \\
\text { - } & \text { Open Access } \\
\text { - } & \text { Biblioteca pública } \\
\text { - } & \text { Desarrollo de } \\
\text { proyectos } \\
\text { - } & \text { Fondos digitales } \\
\text { - Licencias } \\
\text { Creative } \\
\text { Commons }\end{array}$ \\
\hline $\begin{array}{l}\text { Paquetería } \\
\text { básica }\end{array}$ & $\begin{array}{l}\text { - Excell } \\
\text { - Edición de imágenes } \\
\text { - Procesador de palabras } \\
\text { (Word) } \\
\text { - Software de oficina para } \\
\text { presentaciones }\end{array}$ & - Habilidades docentes & $\begin{array}{l}\text { - } \text { Microenseñanza } \\
\text { - Desarrollo de } \\
\text { habilidades docentes } \\
\text { aplicadas a las TIC }\end{array}$ \\
\hline $\begin{array}{l}\text { Habilidades } \\
\text { básicas de } \\
\text { software }\end{array}$ & $\begin{array}{l}\text { - Sistema operativo } \\
\text { - Autoprotección digital } \\
\text { - Descarga e instalación de } \\
\text { programas }\end{array}$ & & \\
\hline
\end{tabular}

Cuadro 1. Implartición de cursos.

Fuente: Elaboración propia.

Cómo se observa, la aplicación y distribución de los diferentes cursos tuvo el objetivo de adquirir y desarrollar competencias y habilidades en diferentes niveles de complejidad, que van desde un nivel básico (que acerca a las cuestiones informáticas), hasta el uso de la computadora y sus principales elementos vinculados con la conectividad. El nivel intermedio amplió los conocimientos de paquetería o software, y permitió desarrollar proyectos de interés tanto para la sociedad, como para el desempeño profesional de cada uno de los bibliotecarios.

Por lo tanto, la mejora se refleja en la capacitación al personal que atiende las bibliotecas del estado. Otro aspecto importante es señalar la alta participación del personal, ya que hay un interés por los temas impartidos, así como 
una inclusión de temas propios del siglo XXI. Finalmente, cabe mencionar que cada uno de los cursos son adaptados a la modalidad en línea y a la presencial.

De manera general, se describen los objetivos aplicados en cada uno de los cursos que formaron parte del modelo de intervención, de acuerdo con cada categoría.

En cuanto a tecnologías de la información, software básico y habilidades básicas de software, se identificaron los diversos componentes que conforman una computadora, así como el uso de los dispositivos de entrada y salida que permiten la interacción entre la persona y la computadora; de igual manera, conocer el concepto de conexión a internet y lo necesario para su uso en la inclusión de gráficos, estilos, desarrollo de presentaciones y el uso de interfaces; para finalmente conocer el sistema operativo, los elementos principales que aparecen en pantalla y comprender los conceptos e iconos, así como el manejo de archivos y carpetas a través de la herramienta de explorador de Windows: crear, borrar mover, copiar, almacenamiento de información en dispositivos externos e introducción a la instalación de programas. En cuanto a los cursos, se dividieron en dos:

- Profesionalizantes. Para adquirir habilidades y conocer conceptos básicos para la planificación de actividades docentes, que permitan generar cursos y material necesario para la transmisión de conocimiento en su comunidad en el área de bibliotecología.

- Habilidades docentes. Para desarrollar habilidades personales para manejar un grupo de personas de forma óptima y dominar las técnicas que permiten interactuar en el proceso de enseñanza-aprendizaje.

\section{Validación del modelo}

En este contexto, se debe mencionar que la importancia de instaurar el modelo de alfabetización por medio de los recursos digitales es una necesidad que exige la colaboración interdisciplinaria de la escuela formal y tradicional, además del aprendizaje informal que se presenta por medio de proyectos y recursos web de instituciones como museos, bibliotecas, institutos de investigación; de alfabetización o de promoción de la ciencia y la cultura, como Vagones de la Ciencia, o el proyecto que encabeza el Instituto para el Desarrollo de la Sociedad del Conocimiento del Estado de Aguascalientes (IDSCEA), o el Instituto para la Educación de las Personas Jóvenes y Adultas (INEPJA) (GEA, 2017a; 2017b), propuestas que implementó el gobierno 
estatal, cuyo objetivo es promover e implementar los servicios de alfabetización y de apoyo a la población, para facilitar el crecimiento social, mediante la interacción de las redes, y de esta manera reducir la brecha digital, de tal manera que se logre una alfabetización integral e incorporarse a la sociedad de la información y del conocimiento.

Más aún, existen esfuerzos a nivel internacional que tienen ese mismo objetivo, como lo proponen Torres y Marzal (2002: 44), quienes subrayan la importancia y la diversidad de las diferentes alfabetizaciones, y a las que se debe apostar por un nuevo paradigma educativo, basado en los recursos multimedia que aportan distintas instituciones, así como en el objetivo de que el ciudadano o usuario adquiera, desarrolle, implemente e integre el aprendizaje por medio de un entorno virtual e hipertextual.

\section{Metodología APLiCAdA}

El modelo de intervención está conformado por siete etapas:

I. Diagnóstico. Se realizó hizo un diagnóstico del número de personal que laboraba en las bibliotecas, así como un análisis de la infraestructura, equipamiento y distribución de las bibliotecas en todo el estado.

II. Identificación. Se identificaron las competencias y habilidades del personal bibliotecario.

III. Diseño. Basados en la información obtenida por el diagnóstico, se diseñaron programas específicos de acuerdo a las diferentes necesidades.

IV. Producción de contenidos. Se diseñaron actividades con contenidos específicos que permitieron desarrollar las habilidades informativas.

V. Capacitación. Se programaron cursos de capacitación de acuerdo a una logística, cuya finalidad fue atender al personal bibliotecario que estaba al frente de cada unidad de información.

VI. Evaluación. De acuerdo a los cursos de capacitación, se obtuvieron resultados que permitieron verificar el alcance y aprovechamiento de los cursos diseñados por los bibliotecarios para cada una de sus comunidades, por lo que la evaluación permitió ver el alcance obtenido en los bibliotecarios.

VII. Certificación. Finalmente, con este esquema de trabajo se pasó a la certificación del modelo propuesto, comprobando que es un modelo viable, flexible, especializado y exitoso para reducir la brecha digital en el entorno de las bibliotecas públicas. 
La puesta en marcha del modelo de intervención se realizó en las instalaciones de la Universidad Autónoma de Aguascalientes (uaa), tanto en los laboratorios de cómputo, como en las aulas de capacitación del sistema bibliotecario de la institución, a todos los bibliotecarios que pertenecían al sistema de bibliotecas públicas del estado de Aguascalientes.

Durante varios meses, los participantes del curso se dedicaron a elaborar materiales didácticos digitales, haciendo uso de la plataforma Moodle, en la que podían subir sus contribuciones para la aplicación con sus usuarios. Finalmente, se dio un seguimiento, el cual permitió medir los alcances paulatinamente logrados gracias a la capacitación. El método de análisis corresponde a partir de los cursos diseñados y de los resultados de aprendizaje de los bibliotecarios.

Los resultados se observan desde dos perspectivas: una enfocada en mencionar que el modelo de intervención ha funcionado, ya que se obtienen habilidades y competencias que avalan a los bibliotecarios con nuevos conocimientos capaces de resolver los retos que presenta este nuevo siglo. También se ha fundamentado la utilidad en el uso de las bibliotecas públicas como un eslabón fundamental para la enseñanza informal hacia la sociedad en general. Y la segunda va encaminada a los resultados obtenidos, pues destaca la impartición de 25 cursos en diferentes temas, que abarcan dos grandes ramas enfocadas a las Tecnologías de la información; la segunda, enfocada a desarrollar las competencias básicas profesionalizantes del perfil bibliotecario.

En los aspectos tecnológicos, se atendieron las competencias básicas para desarrollar habilidades en el uso de computadora, sistemas operativos, navegación por Internet, autoprotección digital, e-comercio, e-comunicación, búsqueda avanzada por navegadores, opciones interactivas digitales, fondos digitales, edición de imágenes, procesador de palabras, hojas de cálculo y diseño, manipulación de imágenes y uso de navegación en la red, en el caso de las competencias básicas profesionalizantes se manejaron cursos como microenseñanza, desarrollo de proyectos, la iniciativa de Open access, las licencias Creative Commons, así como derechos de autor.

Cada curso tuvo la particularidad de atender las necesidades digitales que la población requería, así como adecuarse al modo presencial o en línea, según fuera el caso. A continuación, en la Gráfica 3 se observa la implementación de cursos para capacitar al personal: 


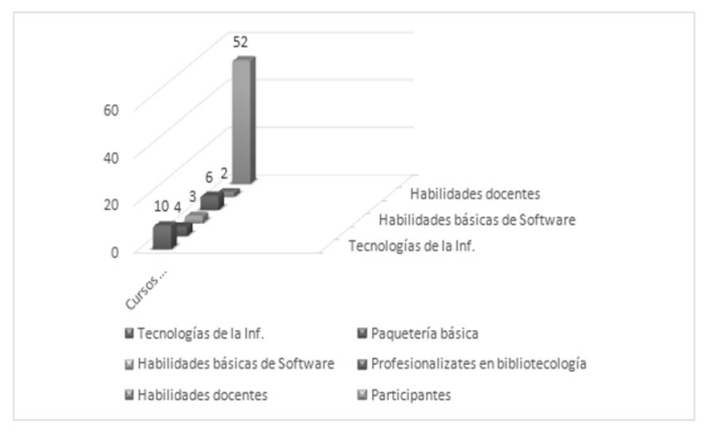

Gráfica 3. Cursos impartidos

Fuente: Elaboración propia.

Por lo tanto, el objetivo del modelo se logró gracias a que se desarrollaron competencias y habilidades informativas, por ejemplo, comprender la estructura del conocimiento, recuperar, analizar, evaluar, integrar y sintetizar la información, así como al uso de dispositivos que les permitió reconocer, utilizar y distinguir la utilidad de los dispositivos, acorde a cada una de las necesidades personales y las de sus usuarios.

En cuanto a la participación, se capacitó a cincuenta y dos personas, repartidas en dos grupos, sin deserciones, pues el 92 por ciento de los participantes concluyó la totalidad los cursos, dando como resultado un producto nuevo que se realizó de manera individual.

Se utilizó un servidor en línea, en el que se alojaron materiales y cursos tomados por los participantes, y que a su vez serán utilizados para los usuarios de las bibliotecas públicas en cada una de las comunidades.

\section{Conclusiones}

Éstas se presentan desde los aspectos cualitativos y cuantitativos, demostrando que se cumple con el objetivo central del modelo en cuestión. En cuanto a los aspectos cualitativos, se observó un avance significativo por parte de los participantes en el dominio de los temas tratados en cada uno de los cursos. De igual manera, los participantes aportaron ideas y propuestas de cómo pueden aplicar, en el contexto de su localidad, las habilidades adquiridas o actualizadas. También se observó una aceptación gradual en cada uno de los cursos, así como una mejora en el nivel de conocimiento y habilidades. 
Los asistentes demostraron interés común en la aplicación de las competencias adquiridas, lo cual fomentó el trabajo colaborativo. Los cursos impartidos fueron de nivel básico, se reforzaron las habilidades y competencias en cada uno de los participantes.

En lo que respecta a los temas expuestos, fueron del interés de los participantes, al ver que son cien por ciento aplicables en el contexto de bibliotecas públicas, sin importar que tan "técnicos" fueran.

Ahora bien, en cuanto a los aspectos cuantitativos, hubo una participación de cincuenta y dos personas en total, divididas en dos grupos. Se observó una asistencia constante a las sesiones, en promedio de 48 participantes de un total de 52, es decir, casi el 92 por ciento de ambos grupos. Se cumplió con la puesta en marcha del cien por ciento de los cursos. No se presentaron casos de baja o deserción entre los asistentes.

Aproximadamente un 35 por ciento de las personas manifestó no poder avanzar en la realización de actividades de tarea en sus localidades, debido a la falta de servicio de Internet en su comunidad. Sin embargo, hacían sus ejercicios de tarea en negocios de Internet público, o bien las realizaban en algún tiempo libre durante las sesiones presenciales en la UUA.

\section{REFERENCIAS}

Álvarez Rodríguez, Francisco Javier, Jaime Muñoz Arteaga, Alma Rosa García Gaona y René Santaolaya Salgado. 2015. Disminución de la brecha digital: casos de aplicación en países de América Latina. México: Pearson.

Area Moreira, Manuel. 2014. "La alfabetización digital y la formación de la ciudadanía del siglo XXI". Rev. de Inv. Educ., vol. 7, no. 3:21-33.

Area Moreira, Manuel. 2002. "Igualdad de oportunidades y nuevas tecnologías.Un modelo educativo para la alfabetización”. Educar, núm. 29:55-65.

Consejo Nacional para Asuntos Bibliotecarios de las Instituciones de Educación Superior (CONPAB-IES). 2012. "Normas para bibliotecas de instituciones de educación superior e investigación”. La Paz, B.C.S.: CONPAB-IES.

Cortés Vera, Jesús, Diana González, Jesús Lau, Ana Lilian Moya, Álvaro Quijano, Lourdes Rovalo y Saúl Soto. 2012. "Normas sobre alfabetización informativa en educación superior. Declaratoria redactada con propuestas de todos los participantes. Tercer encuentro sobre desarrollo de habilidades informativas". La Paz, B.C.S.: CONPAB-IES (col. Normatividad), en <http://www.conpab.org.mx/librosVersionHtml/pdf/Alfabetizacion.pdf $>$.

Dehesa Torres, Abel M., Francisco Javier Álvarez Rodríguez y Jaime Muñoz Arteaga. 2015. Los negocios de Internet públicos en la disminuciòn de la brecha digital. México: Pearson. 
Gill, Philip. 2002. Directrices IFLA/Unesco para el desarrollo del servicio de bibliotecas públicas. México: Conaculta.

Gobierno del Estado de Aguascalientes (GEA). 2017a. "Instituto para el Desarrollo de la Sociedad del Conocimiento del Estado de Aguascalientes (IDSCEA)", en $<$ http://www.aguascalientes.gob.mx/IDSCEA/>.

Gobierno del Estado de Aguascalientes (GEA). 2017b. "Instituto para la Educación de las Personas Jóvenes y Adultas (INEPJA)”, en <http://www.aguascalientes. gob.mx/INEPJA/

Gobierno del Estado de Aguascalientes (GEA). 2016. "Red Estatal de Bibliotecas Públicas”. Aguascalientes: GEA, en <http://www.aguascalientes.gob.mx/temas/ cultura/espacios/bibliotecas/default.aspx>.

IFLA. 1994. "Manifiesto de la IFLA/Unesco sobre la biblioteca pública", en <https:// www.ifla.org/ES/publications/manifiesto-de-la-ifla-unesco-sobre-la-biblioteca-p-blica-1994>, consultada el $1^{\circ}$ de diciembre de 2018.

Muñoz Arteaga, Jaime, José Eder Guzmán Mendoza y Francisco Javier Álvarez Rodríguez. 2014. "Modelos para el desarrollo de las sociedad del conocimiento del estado de Aguascalientes", en Modelos y estrategias para la disminución de la brecha digital en el estado de Aguascalientes. Aguascalientes: UAA, 127-150.

Torres, Nuria y Miguel Ángel Marzal García-Quismondo. 2002. "Alfabetización de la información: recursos electrónicos educativos desde la documentación”. Educación y Biblioteca, núm. 14: 44-51.

Para citar este texto:

González Sandoval, José Luis, Francisco Javier Álvarez-Rodríguez y Jaime Muñoz Arteaga, 2019. "Modelo de intervención para competencias digitales del Programa Bibliotecas Públicas de Aguascalientes”. Investigación Bibliotecológica: archivonomía, bibliotecología e información 33 (80): 155-171.

http://dx.doi.org/10.22201/iibi.24488321xe.2019.80.58012 
